\title{
Who bullies whom at a garden feeder? Interspecific agonistic interactions of small passerines during a cold winter
}

\author{
Katarzyna Wojczulanis-Jakubas • Monika Kulpińska • \\ Piotr Minias
}

Received: 2 February 2015/ Accepted: 23 February 2015/Published online: 11 March 2015

(C) The Author(s) 2015. This article is published with open access at Springerlink.com

\begin{abstract}
Interspecific agonistic interactions are important selective factors for maintaining ecological niches of different species, but their outcome is difficult to predict a priori. Here, we examined the direction and intensity of interspecific interactions in an assemblage of small passerines at a garden feeder, focussing on three finch species of various body sizes. We found that large and mediumsized birds usually initiated and won agonistic interactions with smaller species. Also, the frequency of fights increased with decreasing differences in body size between the participants. Finally, the probability of engaging in a fight increased with the number of birds at the feeder.
\end{abstract}

Keywords Agonistic interactions - Feeder - Finches . Interspecific competition

\section{Introduction}

Aggressive behaviour among animals serves to exclude competitors from limited resources (Stamps 1992). As a result, the distribution of resources is usually unequal, with dominant individuals having priority of access (Gauthreaux 1978; Keddy 2001). By rule of thumb, competition should be the strongest among individuals of the same species, as conspecifics have the most similar demands, which can be intensified in areas with high population densities (Keddy

K. Wojczulanis-Jakubas $(\bowtie) \cdot$ M. Kulpińska Department of Vertebrate Ecology and Zoology, University of Gdańsk, ul. Wita Stwosza 59, 80-308 Gdańsk, Poland e-mail: biokwj@univ.gda.pl

\section{P. Minias}

Department of Teacher Training and Biodiversity Studies, University of Łódź, ul. Banacha 1/3, 90-237 Łódź, Poland
2001). In addition to conspecific, interspecific agonistic interactions can have a substantial impact. In fact, being a critical ecological factor that influences the fitness and survival of birds (Gustafsson 1987; Sasvári et al. 1987), competition among species might be a strong selective force in favour of maintaining the ecological niches of different species (Darlington 1972; Holt 1977; Alatalo et al. 1985; Keddy 2001).

The result of interspecific competition may depend on the morphology of the two participants of the interaction; For example, the different shapes and sizes of avian beaks play an important role in determining hierarchies at interspecific level (Grant 1986). Some studies indicate that body size or body mass could also affect performance in competition (French and Smith 2005). Larger animals usually outcompete smaller ones (Clegg and Owens 2002; Robinson-Wolrath and Owens 2003), although the chance of interaction and the strength of competition is likely to increase along with decreasing difference in body size between the participants of the interaction (Leiquién et al. 2006). Environmental context may also influence competition performance between species. For instance, when preferred food is limited, the foraging niches of two species might overlap to a greater extent than in more desirable circumstances, and species that do not usually interact with each other would start to compete for the same food resources (Oksanen 1987). Moreover, such competing species would compete more intensively as the population density increases (Johnson et al. 2004). For all these reasons, the frequency and results of interspecific aggressive interactions are difficult to predict a priori.

In this study, we examined the direction and intensity of interspecific interactions of birds at a garden feeder during winter in the temperate zone. We focussed on three finch species of similar body and bill structures, but of different 
sizes: large-hawfinch (Coccothraustes coccothraustes, average total body length $=17.5 \mathrm{~cm}$ ), medium-greenfinch (Chloris chloris, $15.5 \mathrm{~cm}$ ) and small-goldfinch (Carduelis carduelis, $13.5 \mathrm{~cm}$ ) (Svensson et al. 2010). Although direct observations of competition between these species have not been reported so far, the three species are expected to interact antagonistically owing to their similar dietary preferences (granivorous), especially in winter, when they gather in mixed flocks (Perea et al. 2014). We expected to find a hierarchical dominance among the species in relation to body size, with larger species outcompeting smaller ones. We also expected to find positive relationships between the intensity of aggressive interactions and the density of birds foraging at the feeder.

\section{Methods}

We conducted the study at a garden bird feeder situated in a suburban area in Rumia, northern Poland $\left(54^{\circ} 22^{\prime} \mathrm{N}\right.$, $\left.18^{\circ} 38^{\prime} \mathrm{E}\right)$. The feeder $\left(21 \times 30 \times 20 \mathrm{~cm}^{3}\right.$; set $2 \mathrm{~m}$ above the ground) was filled with $300 \mathrm{~g}$ of sunflower Helianthus sp. seeds, every morning, starting from late November 2011 until the end of March 2012. Natural fruits of buckthorn Hippophae rhamnoides and rose Rosa rugosa were also placed in close proximity to the feeder from December 2011 onwards.

We recorded the presence of birds and their behaviour with an industrial camera (HS-166 color CCD camera; Mintron, Taiwan) placed $10 \mathrm{~m}$ from the feeder for 15 days (10 February to 1 March, 2012). During daylight hours (ca 06:00-17:00), the camera would record any movement at the feeder and in the near vicinity (radius of $0.5 \mathrm{~m}$ ). The resolution of the camera allowed us to identify the species and note the bird's behaviour. Because of the slight, if any, sexual dimorphism in these species, and the poor light conditions, we could not identify the sexes of birds.

For analysis, we divided the footage into 15-min sessions of each hour of recording. Thus, in total we examined 165 sessions covering $41.3 \mathrm{~h}$. The occurrence of any agonistic interaction among the birds at the feeder during each session was noted. If interaction occurred, the following parameters were noted: (a) the species of birds involved, the initiator and the sufferer being distinguished, (b) the intensity of the behaviour and (c) the result of the interaction for the sufferer. A bird was considered the initiator if it was the first one to perform agonistic behaviour. The intensity of each interaction was categorised as threatening if the initiator presented a threatening posture (beak open, and/or feathers bristling, and/or wings/head lowered) but without physical contact. If any physical contact did occur, the interaction was considered a fight. A participant making threatening and fighting postures was treated as the loser if it retreated from the place or left the feeder area while the other bird stayed; the one that stayed was thus considered the winner. Interactions in which no apparent winner or loser could be discerned were treated as unsettled. The number of birds present at the feeder, i.e. the density, was recorded at the moment of the interaction. A total of 1512 interactions were recorded.

\section{Data analysis}

Apart from the three finch species, four other species participated in aggressive interactions more than once (brambling Fringilla montifringilla, great tit Parus major, siskin Carduelis spinus and blue tit Cyanistes caeruleus), so we included them in the analyses. We assessed the structural body size of the interacting species on the basis of total body length (the average of the two extreme ranges given in Svensson et al. 2010).

We used Fisher's exact test to examine the proportion of agonistic interactions (threatening and fighting combined) of the three finches with other bird species, when any of the three was involved. We used the generalised linear model (GLM) with binominal errors and a logit link function to analyse the effects of the structural body size of sufferers and the density of feeding birds on fight occurrence during agonistic interactions and their consequences in the three finches. We excluded intraspecific interactions and those unsettled from the analyses. The significance of the independent variables was assessed using the likelihood ratio (chi-square) test. We present all values as mean \pm standard error (SE) and performed all statistical analyses with Statistica 10.0 (StatSoft, USA) and JMP Pro 10 (SAS Institute 2012).

\section{Results}

Hawfinches were mostly the initiators of agonistic interactions with other species (Fisher's exact tests, all $p<0.01$; Fig. 1a). Consequently, the probability of engaging in a fight during an agonistic interaction initiated by hawfinches did not depend on the body size of sufferers (GLM, $\chi^{2}=0.09, n=238, p=0.77$ ). Hawfinches were also the winners in almost all interactions with other species (Fisher's exact tests, all $p<0.001$; Fig. 1b).

Greenfinches were mostly the initiators of interactions with other species (Fisher's exact test, all $p<0.001$ ). Exceptions were: (1) interactions with hawfinches, where greenfinches were mostly the sufferers (Fisher's exact test, $p<0.001$; Fig. 1a) and (2) interactions with great tits and blue tits, where the frequencies of interactions initiated and suffered were similar (both $p>0.05$ ). The probability of 
Fig. 1 Proportion of agonistic interactions (threats and fights combined) among the species: a initiated by hawfinches (black), greenfinches (grey) and goldfinches (white); b won by the three finch species. Numbers above bars denote the number of recorded interactions for a given pair of species. Species ordered by size (total body length). Only sample sizes are shown for intraspecific interactions
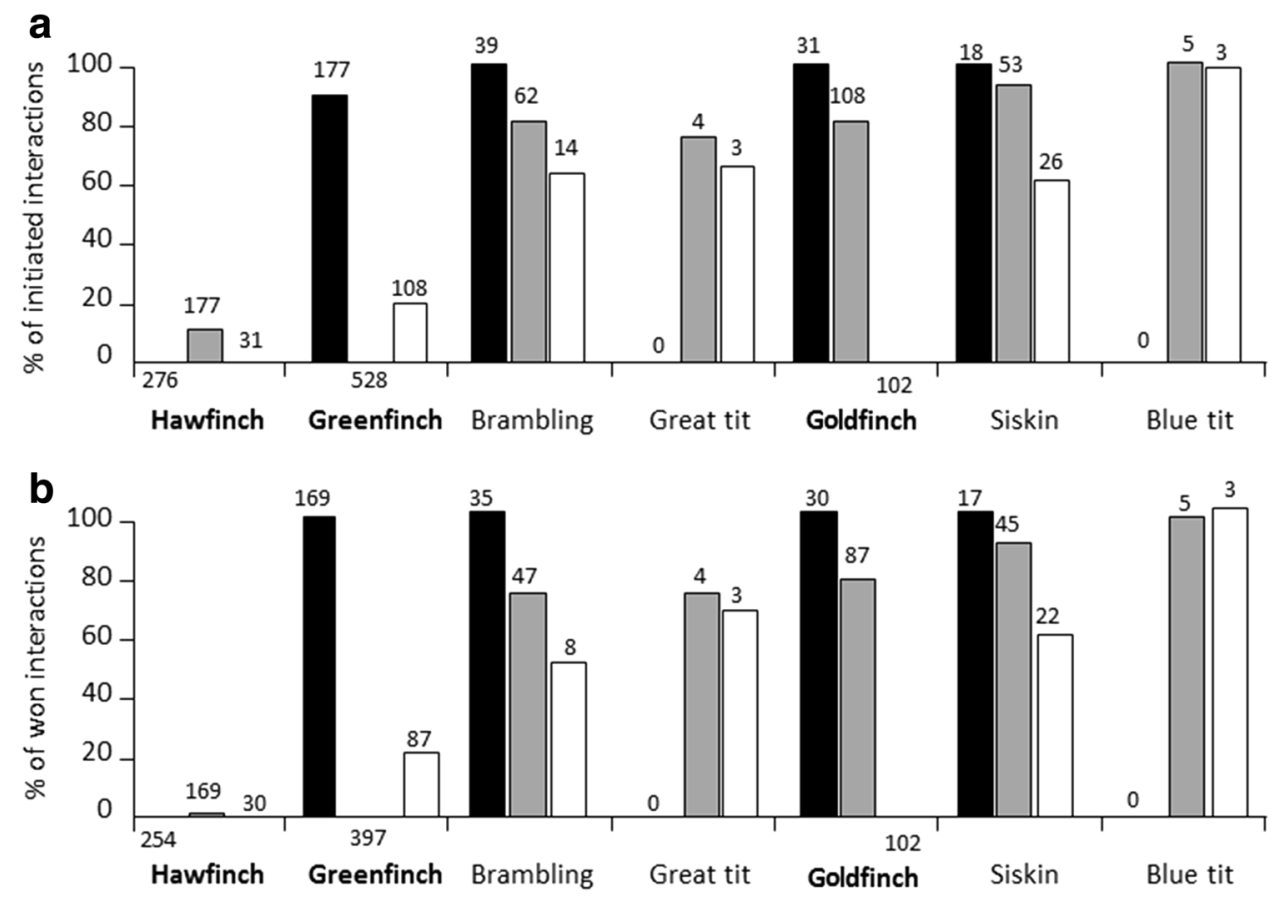

greenfinches engaging in a fight during agonistic interactions that they initiated increased with the body size of the sufferers (GLM, $\quad \chi^{2}=6.57, \quad n=168, \quad p=0.010$, $\beta=0.24 \pm 0.09)$. Consequently, when initiating an interaction with individuals of smaller body size, greenfinches tended to confine their agonistic behaviour to threats. Greenfinches were the winners in most of the interactions (Fisher's exact tests, all $p<0.01$; Fig. 1b), except for the ones with hawfinches $(p<0.001)$. In interactions with great tits and blue tits, the frequencies of winning and losing were similar (Fisher's exact test, $p=0.42$ and $p=0.17$, respectively; Fig. $1 \mathrm{~b}$ ). The probability of greenfinches winning agonistic interactions depended largely on the body size of the sufferers (GLM, $\left.\chi^{2}=42.28, n=357, p<0.001\right)$. The probability of winning was higher in threatening interactions than in fighting (GLM, $\chi^{2}=4.91, n=357, p=0.027$ ), but the effect was not statistically significant after accounting for the body size of the competitors $\left(\chi^{2}=2.32, n=357, p=0.13\right)$.

Goldfinches were the sufferers in interactions with the two larger finches and were sufferers as frequently as initiators in interactions with other species (Fisher's exact test, all $p>0.05$; Fig. 1a). Goldfinches were equally likely to engage in fights with smaller and larger species when initiating interactions (GLM, $\chi^{2}=0.001, \quad n=38$, $p=0.97)$. In the interactions with other species, goldfinches were winners and losers with similar frequencies (Fisher's exact tests, all $p>0.24$; Fig. 1b). In interactions with hawfinches and greenfinches, goldfinches were usually losers $(p<0.001)$. The probability of winning did not depend on the size of the competitor (GLM, $\chi^{2}=1.09$, $n=153, p=0.30)$.

The density of feeding birds affected the intensity of agonistic behaviour in all three species. The probability of engaging in a fight increased with the number of feeding birds (GLM, hawfinch: $\chi^{2}=9.10, n=252 p=0.003, \beta=0.38$ \pm 0.13 ; greenfinch: $\chi^{2}=23.43, \quad n=357, \quad p<0.001$, $\beta=0.34 \pm 0.07 ; \quad$ goldfinch: $\quad \chi^{2}=12.75, \quad n=153$, $p<0.001, \beta=0.38 \pm 0.11)$. As indicated by the odds ratios [hawfinch: 1.46, $95 \%$ confidence interval (CI) 1.14-1.90; greenfinch: $1.40,95 \%$ CI 1.22-1.62; goldfinch: $1.46,95 \%$ CI 1.18-1.84], the probability of engaging in a fight during an agonistic interaction increased 1.40-1.46 times for each additional member of the respective feeding flock, depending on the species. Greenfinches were also more likely to initiate agonistic interactions towards species of larger sizes while feeding in higher densities $\left(F_{1,166}=6.42, p=0.012\right.$, $\beta=0.16 \pm 0.07$; Fig. $2 \mathrm{~b}$ ), although no such relationship was found in hawfinches $\left(F_{1,236}=0.00, p=1.00\right.$; Fig. $\left.2 \mathrm{a}\right)$ or goldfinches $\left(F_{1,36}=0.36, p=0.55\right.$; Fig. $\left.2 \mathrm{c}\right)$.

\section{Discussion}

This study shows that some species of finches are competitively superior to others, and this competitive hierarchy seems to be linked to body size. The large and medium-sized finches usually initiated and won agonistic interactions with smaller species. Moreover, the intensity of the interactions was also driven by the body size of the participants, with the 

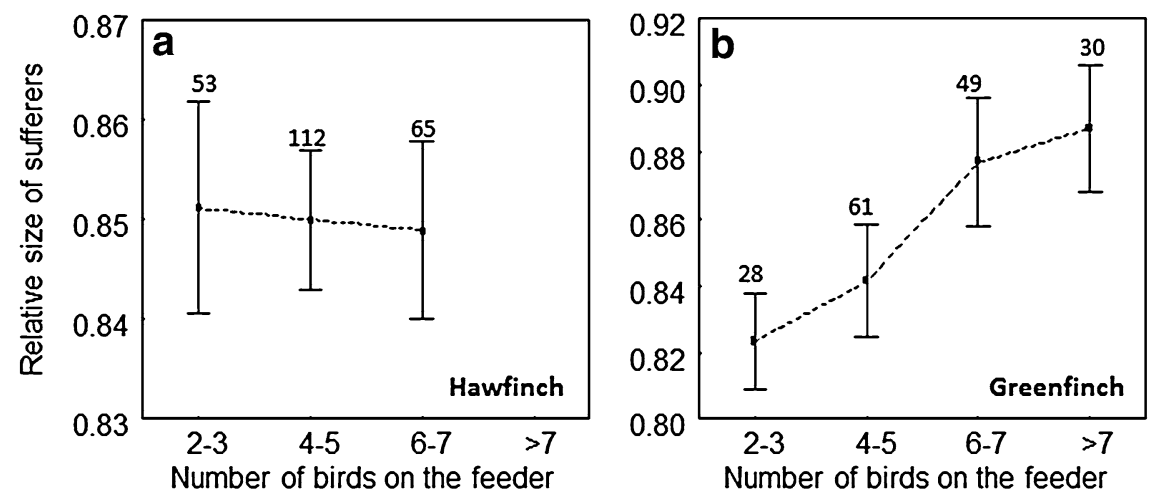

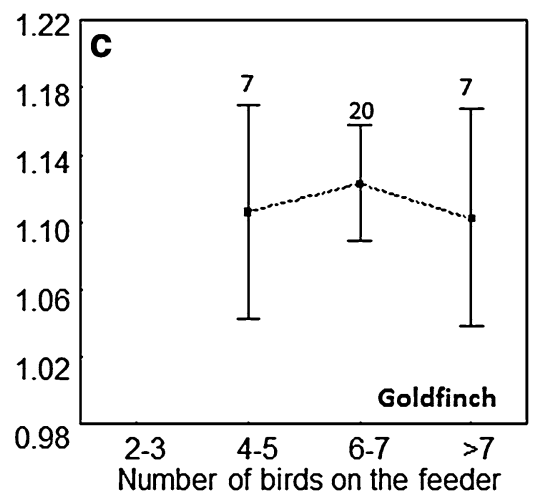

Fig. 2 Relative size of sufferers to those of initiators in aggressive interactions, initiated by $\mathbf{a}$ hawfinches, $\mathbf{b}$ greenfinches and $\mathbf{c}$ goldfinches in relation to the number of birds at the feeder (mean $\pm \mathrm{SE}$ ). In each case, the size of the initiator is equal to 1 . The numbers of birds

frequency of fighting increasing with decreasing difference in body size between the participants. This is consistent with the results of some other studies examining the relationships between animal body size and interspecific competition (e.g. Wilson 1975; Dhondt and Eyckerman 1980; RobinsonWolrath and Owens 2003). As such, our study supports the hypothesis that body size would be an important factor affecting the general performance of animals and ecological networks (Woodward et al. 2005).

The probability of winning agonistic interactions initiated by hawfinches and goldfinches did not depend on the size of sufferers. In contrast, greenfinches displayed a higher probability of winning and confined their agonistic behaviour to threats when interacting with smaller species. These interspecific differences might be attributed to limited opportunity, rather than the lack of the effect of body size: as the hawfinch was the largest and the goldfinch was almost the smallest species visiting the feeder, all sufferers of interactions were smaller than initiator hawfinches and larger than initiator goldfinches. Therefore, the results for the hawfinch and goldfinch should be treated with caution.

Intensified aggressive interactions between individuals are in general associated with limited resources (Moore and Yong 1991; Dubois 2003). In our study, although the amount of food at the feeder remained constant during the whole study period, the amount of food per capita should decrease when the number of visitors to the feeder increased. As a consequence, the probability of engaging in a fight increased 1.40-1.46 times for each additional member of the foraging flock. This suggests that restricted food resources may indeed drive competition between the species.

Alternatively, the increasing intensity of agonistic behaviours with increasing density of birds at the feeder may be the result of a simple violation of individual distance. On condition that individual distances are disturbed, agonistic behaviours are more likely to occur (Hall 1966). Studies to date indicate that the principle of individual distance operates at the feeder were grouped into four levels; the highest level $(>7)$ for the hawfinch, and the smallest (2-3) for the goldfinch are not shown because of the small sample sizes ( $n=8$ and $n=4$, respectively)

in various groups of animals, including birds (e.g. Hinde 1956; Slotow 1996; Nephew and Romero 2003). It is plausible, therefore, that individual distance violation intensified along with the increasing number of birds at the feeder independently of, or in combination with, the amount of resources.

The present results were obtained at a garden feeder, i.e. in highly artificial conditions. The recorded frequency of agonistic encounters per se is therefore unlikely to be observed in nature. However, the revealed relationships, such as the body-size-dependent outcome, should be valid in natural conditions. Other findings, such as the increase in intensity of aggressive interactions under conditions of high bird densities, also seem to be valid, despite the artificial circumstances. It has been found in other species that the outcome of interspecific competition during winter may reflect the situation during the breeding season (Dhondt and Eyckerman 1980). Thus, it is possible that the dominance hierarchy between finches at a garden feeder during winter accounts for the spatial segregation of species while they are foraging together (Perea et al. 2014).

Acknowledgments We are grateful to Izabela Kulaszewicz for making the feeder available for the study and operating the camera. We also thank Jakub Wietrzykowski for help with video analyses, and Dariusz Jakubas for inspiring discussions. Last but not least, thanks go to Zack Bateson and Peter Seen for help with English usage, and to reviewers and editors for kind and helpful suggestions that helped to improve the manuscript.

Open Access This article is distributed under the terms of the Creative Commons Attribution License which permits any use, distribution, and reproduction in any medium, provided the original author(s) and the source are credited.

\section{References}

Alatalo RV, Gustafsson L, Linden M, Lundberg A (1985) Interspecific competition and niche shifts in tits and the goldcrest: an experiment. J Anim Ecol 54:977-984 
Clegg SM, Owens IPF (2002) The 'island rule' in birds: medium body size and its ecological explanation. Proc R Soc Lond B 269:1359-1365

Darlington PJ Jr (1972) Competition, competitive repulsion, and coexistence. Proc Natl Acad Sci USA 69:3151-3155

Dhondt AA, Eyckerman R (1980) Competition between the great tit and the blue tit outside the breeding season in field experiments. Ecology 61:1291-1296

Dubois F (2003) Resource defense in a group-foraging context. Behav Ecol 14:2-9

French AR, Smith TB (2005) Importance of body size in determining dominance hierarchies among diverse tropical frugivores. Biotropica 37:96-101

Gauthreaux SA Jr (1978) The ecological significance of behavioral dominance. In: Bateson PPG, Klopfer PH (eds) Perspectives in ethology. Plenum, New York, pp 17-54

Grant PR (1986) Ecology and evolution of Darwin's finches. Princeton University, Princeton

Gustafsson L (1987) Interspecific competition lowers fitness in collared flycatchers Ficedula albicollis: an experimental demonstration. Ecology 68:291-296

Hall ET (1966) The hidden dimension. Anchor Books Editions, America

Hinde A (1956) The biological significance of the territories of birds. Ibis 92:340-369

Holt RD (1977) Predation, apparent competition, and the structure of prey communities. Theor Pop Biol 12:197-229

Johnson CA, Grant JWA, Giraldeau L-A (2004) The effect of patch size and competitor number on aggression among foraging house sparrows. Behav Ecol Sociobiol 15:412-418

Keddy PA (2001) Competition. Kluwer, Dordrecht
Leiquién E, de Boer WF, Cleef A (2006) Influence of body size on coexistence of bird species. Ecol Res 22:735-741

Moore FR, Yong W (1991) Evidence of food-based competition among passerine migrants during stopover. Behav Ecol Sociobiol 28:85-90

Nephew C, Romero LM (2003) Behavioral, physiological, and endocrine responses of starlings to acute increases in density. Horm Behav 44:222-232

Oksanen L (1987) Interspecific competition and the structure of bird guilds in boreal Europe: the importance of doing fieldwork in the right season. Trends Ecol Evol 2:376-379

Perea E, Venturas M, Gil L (2014) Seed predation on the ground or in the tree? Size-related differences in behaviour and ecology of granivorous birds. Acta Ornithol 49:119-130

Robinson-Wolrath SI, Owens IPF (2003) Large size in an islanddwelling bird: intraspecific competition and the dominance hypothesis. J Evol Biol 16:1106-1114

Sasvári L, Török J, Tóth L (1987) Density dependence between three competitive bird species. Oecologia 72:127-130

Slotow R (1996) Aggression in white-crowned sparrows: effects of distance from cover and group size. Condor 98:245-252

Stamps JA (1992) Simultaneous versus sequential settlement in territorial species. Am Nat 139:1070-1088

Svensson L, Mullarney K, Zetterström D, Grant PJ (2010) Collins bird guide. Harper Collins, UK

Wilson DS (1975) The adequacy of body size as a niche difference. Am Nat 970:769-784

Woodward G, Ebenman B, Emmerson M, Montoya JM, Olesen JM, Valido A, Warren PH (2005) Body size in ecological networks. Trends Ecol Evol 20:402-409 